\title{
EFEITO DA CHUVA SOBRE A TENACIDADE E EFICIÊNCIA DE FUNGICIDAS CÚPRICOS ASSOCIADOS AO ÓLEO VEGETAL NO CONTROLE DA FERRUGEM DO CAFEEIRO
}

\author{
SILVÂNIA H. F. OLIVEIRA' ${ }^{1}$, JOSÉ MARIA F. SANTOS ${ }^{2} \&$ SYLVIA D. GUZZO $^{2}$ \\ ${ }^{1}$ Centro de Sanidade Vegetal, Instituto Biológico, Cx. Postal 70, CEP 13001-970, Campinas, SP, \\ e-mail: oliveirasi@biologico.br; ${ }^{2}$ Centro de Sanidade Vegetal, Instituto Biológico, \\ Av. Conselheiro Rodrigues Alves, 1252, CEP 04014-002, São Paulo, SP
}

(Aceito para publicação em 19/08/2002)

Autor para correspondência: Silvânia H. F. Oliveira

OLIVEIRA, S.H.F., SANTOS, J.M.F. \& GUZZO, S.D. Efeito da chuva sobre a tenacidade e eficiência de fungicidas cúpricos associados ao óleo vegetal no controle da ferrugem do cafeeiro. Fitopatologia Brasileira 27:581-585. 2002.

\section{RESUMO}

A ação da chuva sobre a tenacidade e eficiência de diferentes fungicidas cúpricos isolados ou associados ao óleo vegetal no controle da ferrugem (Hemileia vastatrix) do cafeeiro (Coffea arabica) foi avaliada no presente trabalho. Óxido cuproso, hidróxido de cobre e oxicloreto de cobre, na proporção de $0,3 \%$ do ingrediente ativo, foram pulverizados em mudas de café $24 \mathrm{~h}$ antes da inoculação dos urediniosporos. Após $60 \mathrm{~min} \mathrm{da}$ pulverização, metade das mudas recebeu chuva induzida de 20 mm durante $6 \mathrm{~min}$, repetidas quatro vezes com intervalos de uma semana. A outra metade não recebeu água por aspersão. O fungicida óxido cuproso sem adição do óleo foi mais eficiente do que com adição do óleo. Com o fungicida hidróxido de cobre ocorreu o inverso, enquanto que com o oxicloreto de cobre não se observou a ação do óleo. A presença ou não da chuva, não interferiu significativamente na incidência e severidade da ferrugem, sugerindo que os fungicidas testados apresentam certa resistência à ação da chuva, com boas características de aderência e/ou persistência na superfície foliar do cafeeiro. A mistura do oxicloreto de cobre ao óleo vegetal com ou sem chuva reduziu a germinação média de esporos para $13,51 \%$, sendo significativamente diferente da testemunha com germinação média de $41,29 \%$, o que indica a sua ação erradicante. Destacou-se ainda o óxido cuproso sem óleo na ausência de chuva por erradicar o fungo, mas que na presença de chuva não diferiu da testemunha.

Palavras-chave adicionais: Hemileia vastatrix, precipitação simulada, casa de vegetação.

ABSTRACT

Effect of rain on tenacity and efficiency of fungicides associated with vegetable oil in the control of rust coffee disease

\begin{abstract}
This work was carried out to verify the effect of precipitation on different copper fungicides application and its efficiency for controling coffee (Coffea arabica) rust caused by Hemileia vastatrix, in the presence or absence of vegetable oil. Cuprous oxide, hydroxide of copper and copper oxicloride, in the proportion of $0.3 \%$ of the active ingredient, were sprayed on coffee plants $24 \mathrm{~h}$ before inoculation with urediniospores. $60 \mathrm{~min}$ after spraying, half of the plants received $20 \mathrm{~mm}$ of rain for $6 \mathrm{~min}$; repeated four times at weekly intervals. The other half of the plants did not receive water. Cuprous oxide was more efficient without oil than with oil, contrasting with the results obtained for hydroxide of copper. Adding oil did not improve copper oxicloride action.
\end{abstract}

\section{INTRODUÇÃO}

O aparecimento da ferrugem (Hemileia vastatrix Berk. \& Br.) do cafeeiro (Coffea arabica L.) no Brasil, em 1970, doença que já havia devastado a cafeicultura de vários países, promoveu uma revolução nas práticas culturais das lavouras brasileiras, modernizando e aperfeiçoando os métodos de condução da cultura.
Rain was not observed to make any significant difference in the incidence and severity of coffee rust among the plants. This suggests that, besides copper compounds being contact products, they showed a certain resistance to the action of the rain, with good characteristics of adherence and/or persistence on the surface of the coffee leaves. The mixture of copper oxicloride with oil, independent of the presence of rain, was efficient in reducing the viability of the spores to $13.5 \%$, indicating the erradication action of this treatment, while the test treatment showed $41.29 \%$. Cuprous oxide with no oil in the absence of the rain also provided no sporulation action, but did not differ from the test plots where rain was present.
O manejo inadequado dos cultivos e condições de ambiente favoráveis de alta umidade e temperatura entre 21 e $25{ }^{\circ} \mathrm{C}$ propiciam a ocorrência da ferrugem (Zambolim et al., 1985; Kushalappa \& Eskes, 1989), que em ataques severos promovem grande desfolha com perdas de até $50 \%$ de produtividade e na qualidade, em anos com deficit hídrico acentuado (Godoy et al., 1997; Zambolim \& Vale, 2000). A pulverização com fungicidas cúpricos representa um 
dos métodos de controle mais tradicionais contra a doença. No entanto, recomenda-se também o uso de fungicidas ditiocarbamatos que são menos eficientes por apresentarem baixo efeito residual e serem facilmente laváveis pela chuva, e os sistêmicos (triazóis) usados por aspersão nas folhas e os granulados, via solo (Instituto Brasileiro do Café, 1979; Zambolim et al., 1990; Matiello, 1991). Os cúpricos além de serem eficientes também contra outras doenças, mostram efeito nutricional favorável ao desenvolvimento do cafezal (Almeida \& Matiello, 1999; Zambolim \& Vale 2000). Na sua utilização, é necessária a realização de um número relativamente grande de pulverizações, principalmente em período chuvoso do ano e nem sempre o produtor consegue realizá-las em número adequado. Em alguns locais adotamse outras medidas de controle como a pulverização de fungicidas sistêmicos (triadimenol, cyproconazole) nas folhas e a mistura de fungicidas + inseticidas via solo (triadimenol + dissulfoton), sem efeitos negativos sobre a qualidade e com melhoria do padrão de controle fitossanitário das lavouras (Souza, 1996). O uso de inseticida + fungicida via solo por muitos anos consecutivos em safras de alta e de baixa carga da lavoura não é uma medida adequada, pois afeta a sustentabilidade da lavoura (Zambolim \& Vale, 2000).

As características de um fungicida são fatores determinantes dentro de uma série de variáveis que determinam a efetividade de um produto. A quantidade de produto que adere à folha durante a pulverização e a quantidade de material que permanece na folha após a ação de intempéries são os principais fatores que determinam a quantidade de resíduo ativo nas superfícies foliares para um efetivo controle dos fitopatógenos (Rich, 1954). A chuva é considerada como o maior depreciador de depósitos fungicidas sobre a superfície foliar. Weber et al. (1937) demonstraram que uma única chuva de característica forte remove muito mais fungicida da superfície foliar do que várias chuvas fracas de mesmo volume.

A estratégia do uso de aditivos à calda fungicida pode melhorar a ação de certos fungicidas sensíveis à ação de intempéries, por propiciar melhor aderência à superfície foliar e ainda, elevar a sua persistência (tenacidade), principalmente sob ação da chuva (Suheri \& Latin, 1991).

Devido à importância dos fungicidas cúpricos na cafeicultura e pelo fato de os fungicidas de contato apresentarem problemas de serem lavados pela precipitação, este trabalho objetivou avaliar a ação de chuva induzida e seqüenciada sobre a tenacidade e eficiência de diferentes formulações de produtos à base de cobre, em associação ou não a óleo vegetal, no controle da ferrugem do cafeeiro e na viabilidade dos esporos do fungo.

\section{MATERIAL E MÉTODOS}

O experimento foi conduzido em condições de casade-vegetação utilizando-se mudas de café cv. Mundo Novo, com cerca de cinco a seis pares de folhas definitivas, cultivadas em sacos plásticos de três litros.
Inicialmente as mudas foram pulverizadas até o ponto de escorrimento, com o auxílio de um pulverizador costal, tendo como propelente o $\mathrm{CO}_{2}$, com os diferentes tratamentos fungicidas cúpricos. A dosagem dos fungicidas óxido cuproso, hidróxido de cobre e oxicloreto de cobre foi determinada considerando-se a concentração de $0,3 \%$ do ingrediente ativo (cobre) para cada produto, correspondendo a $1.200 \mathrm{~g}$ de i.a. em um volume de 4001 de água por hectare.

O preparo da calda com adição de óleo iniciou-se com a adição de água ao fungicida na proporção de 1:2 para formar uma pasta; em seguida, adicionou-se o óleo vegetal (Natur'l Óleo) a 0,3\% e, por fim, completou-se o volume com água.

Após $60 \mathrm{~min}$ da pulverização, tempo considerado suficiente para a secagem das folhas, metade das mudas, ou seja, 70 plantas (dez por tratamento) receberam chuva de 20 $\mathrm{mm}$ durante $6 \mathrm{~min}$, repetindo-se por mais três vezes, semanalmente, totalizando quatro induções de chuvas seqüenciadas. A simulação de chuva foi realizada por meio de um protótipo experimental para aplicação de chuva controlada de acordo com a pressão e volume dos bicos injetores. A sequiência de irrigação objetivou verificar a resistência dos produtos à ação da chuva, denominada de tenacidade ou persistência. A outra metade das mudas não receberam nenhuma chuva ou irrigação por aspersão.

Decorridas 24 h da pulverização dos fungicidas cúpricos, as mudas foram inoculadas com os uredioniosporos de $H$. vastatrix na concentração de $2 \mathrm{mg}$ de esporos/ml de água. Posteriormente, as mudas foram mantidas no escuro por $72 \mathrm{~h}$ em câmara climatizada, com temperatura de $22 \pm 1{ }^{\circ} \mathrm{C}$ e umidade relativa elevada $(80 \pm 2 \%)$ para favorecer a germinação dos esporos.

As avaliações foram realizadas aos 30, 45 e 60 dias após a inoculação (DAI), baseando-se na incidência (número de folhas com pústulas) e severidade (porcentagem de área foliar afetada), considerando-se uma escala de 0 a $50 \%$ de infecção. Foram considerados os pares de folhas marcados na altura mediana da planta, desprezando-se os ramos novos que não recebeu as pulverizações.

O delineamento experimental foi de blocos ao acaso com 14 tratamentos e dez repetições, representadas cada uma por uma planta.

Os urediniosporos produzidos nas folhas inoculadas em cada muda foram coletados com auxílio de um pincel aos 62 DAI, isoladamente para cada tratamento, e avaliados quanto à capacidade de germinação in vitro. Estes foram colocados em placas escavadas e incubados em condições adequadas para a germinação, por um período de $6 \mathrm{~h}$, quando foram imediatamente avaliados e contados, considerando-se os urediniosporos com tubo germinativo. Foram utilizadas quatro repetições (placas) por tratamento. $\mathrm{O}$ delineamento experimental foi inteiramente casualizado.

\section{RESULTADOS E DISCUSSÃO}

Todos os fungicidas cúpricos foram eficientes no controle da ferrugem, estando de acordo com os trabalhos 
Efeito da chuva sobre a tenacidade e eficiência de fungicidas...

realizados desde o início do seu uso na década de 70 (IBC, 1979; Zambolim et al. 1985) até os dias atuais (Almeida \& Matiello, 1999; Santinato et al., 1999; Almeida \& Matiello, 2000). Não houve diferenças entre as testemunhas com chuva e sem chuva nas diferentes avaliações realizadas, mostrando que a quantidade de chuva recebida nas plantas sem tratamento químico não afetou o desenvolvimento final da doença.

Na primeira avaliação das mudas, aos $30 \mathrm{DAI}$, o fungicida óxido cuproso sem óleo e na ausência de chuva apresentou o maior nível de controle da ferrugem, acima de $90 \%$, seguido do mesmo produto na presença de chuva (79\%), de hidróxido de cobre + óleo na presença ou não de chuva (cerca de $80 \%$ ) e de oxicloreto de cobre na ausência de chuva (cerca de 80\%). Além desses, apresentaram eficiência moderada, variando entre $59 \%$ e $69 \%$, o fungicida oxicloreto de cobre na presença de chuva e a sua mistura com óleo na presença ou ausência de chuva (Tabela 1).

Na segunda avaliação, aos 45 DAI, os resultados de eficiência de controle foram semelhantes à avaliação anterior, com níveis de controle variando de 63,5\% até 91,9\% para os melhores tratamentos. Somente hidróxido de cobre sem óleo, independente da chuva, e óxido cuproso + óleo na ausência de chuva não diferiram da testemunha (Tabela 1).

$\mathrm{Na}$ terceira avaliação, aos $60 \mathrm{DAI}$, novamente óxido cuproso na ausência de chuva apresentou nível de controle elevado $(94,9 \%)$, seguido dos mesmos tratamentos citados nas avaliações anteriores, com níveis variando entre $72,8 \%$ a $88,6 \%$. Não apresentaram boa eficiência os tratamentos óxido cuproso + óleo e hidróxido de cobre, independentes da chuva, e oxicloreto de cobre + óleo na presença de chuva (Tabela 1).
Quanto à ação da chuva, não houve diferenças significativas de incidência e severidade da ferrugem para a maioria dos tratamentos que recebeu e que não recebeu a chuva simulada, indicando boa persistência dos cúpricos. No entanto, óxido cuproso + óleo apresentou um melhor desempenho na presença de chuva, possivelmente pela melhor distribuição na superfície foliar. A persistência variou em função do óleo vegetal e o tipo de formulação de cobre associado. Em geral, o óxido cuproso foi melhor sem óleo do que com óleo, enquanto que para o hidróxido de cobre ocorreu o inverso. Oxicloreto de cobre não sofreu ação significativa do óleo (Figuras 1, 2 e 3).

Quanto à germinação in vitro dos urediniosporos de $H$. vastatrix, observa-se que o tratamento óxido cuproso sem chuva impediu completamente a germinação, e oxicloreto de cobre com óleo reduziu a viabilidade dos esporos (índice médio de 13,5\%), independente da presença de chuva, indicando que estes tratamentos possuem ação antiesporulante. Os demais tratamentos fungicidas, com germinação média de $42,0 \%$, não diferiram da testemunha (Tabela 2).

Os trabalhos relacionados à ação de chuva na efetividade de fungicidas são escassos. Oliveira et al. (1995) e Tofoli et al. (1996) verificaram baixa persistência do fungicida mancozeb em trigo (Triticum aestivum L.) e em tomate (Lycopersicon esculentum Mill), respectivamente, sob condições de chuva artificial, com incremento da ação de controle pela adição de óleo vegetal ao fungicida. Em cafeeiro, Tofoli et al (1998) observaram que a ocorrência de chuva induzida de $30 \mathrm{~mm}$ aos 60 e 120 min após a pulverização promoveu decréscimo da efetividade de oxicloreto de cobre no controle da ferrugem. No presente trabalho, as diferenças deste produto na ausência de chuva e

TABELA 1 - Efeito da chuva na porcentagem (\%) de controle da ferrugem (Hemileia vastatrix), avaliada através da severidade, em mudas de cafeeiro (Coffea arabica) tratadas com diferentes fungicidas cúpricos na presença ou ausência de óleo vegetal

\begin{tabular}{lcccc}
\hline \hline Tratamento & Chuva & 30 DAI ** & 45 DAI** & 60 DAI** \\
\hline 1- testemunha & SC & - & - & - \\
2- testemunha & CC & - & - & - \\
3- óxido cuproso & SC & $93,8 \mathrm{e}$ & $91,9 \mathrm{e}$ & $94,9 \mathrm{e}$ \\
4- óxido cuproso & $\mathrm{CC}$ & $79,0 \mathrm{de}$ & $77,4 \mathrm{de}$ & $87,0 \mathrm{de}$ \\
5- óxido cuproso + óleo & $\mathrm{SC}$ & $13,6 \mathrm{abc}$ & $14,4 \mathrm{abc}$ & $50,0 \mathrm{abcd}$ \\
6- óxido cuproso + óleo & $\mathrm{CC}$ & $44,4 \mathrm{abcd}$ & $58,3 \mathrm{bcd}$ & $63,7 \mathrm{abcd}$ \\
7- hidróxido de cobre & $\mathrm{SC}$ & $6,2 \mathrm{abc}$ & $19,8 \mathrm{abc}$ & $6,3 \mathrm{a}$ \\
8- hidróxido de cobre & $\mathrm{CC}$ & $0 \mathrm{a}$ & $8,7 \mathrm{ab}$ & $10,3 \mathrm{abc}$ \\
9- hidróxido de cobre + óleo & $\mathrm{SC}$ & $82,7 \mathrm{de}$ & $80,2 \mathrm{de}$ & $88,6 \mathrm{de}$ \\
10- hidróxido de cobre + óleo & $\mathrm{CC}$ & $80,2 \mathrm{de}$ & $81,7 \mathrm{de}$ & $78,8 \mathrm{de}$ \\
11- oxicloreto de cobre & $\mathrm{SC}$ & $82,7 \mathrm{de}$ & $75,7 \mathrm{de}$ & $83,5 \mathrm{de}$ \\
12- oxicloreto de cobre & $\mathrm{CC}$ & $59,2 \mathrm{bcde}$ & $63,5 \mathrm{cde}$ & $72,6 \mathrm{bcde}$ \\
13- oxicloreto de cobre + óleo & $\mathrm{SC}$ & $67,9 \mathrm{cde}$ & $67,6 \mathrm{de}$ & $72,8 \mathrm{cde}$ \\
14- oxicloreto de cobre + óleo & $\mathrm{CC}$ & $69,1 \mathrm{cde}$ & $66,9 \mathrm{cde}$ & $66,4 \mathrm{abcde}$ \\
\hline CV\% & & 38,0 & 32,0 & 34,5 \\
\hline
\end{tabular}

$* \mathrm{SC}=$ sem chuva; $\mathrm{CC}=$ com chuva

**Médias seguidas pela mesma letra, na vertical, não diferem entre si pelo teste Tukey a 5\% de probabilidade. 


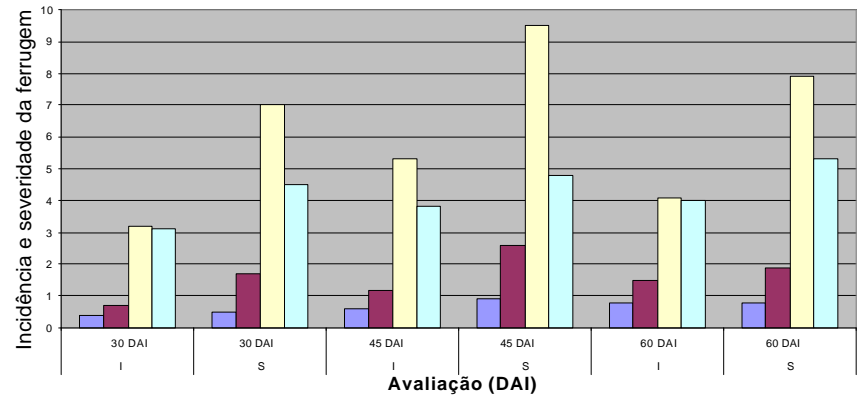

$\square$ Óxido cuproso s/chuva

口Óxido cuproso+óleo s/chuva

口 Óxido cuproso c/chuva

$\square$ Óxido cuproso+óleo c/chuva

FIG. 1 - Comparativo de eficiência com fungicida a base de óxido cuproso, com e sem adição de óleo, na incidência (I) e severidade (S) da ferrugem (Hemileia vastatrix) em mudas de cafeeiros (Coffea arabica) tratadas, sob a influência ou não de chuva simulada.

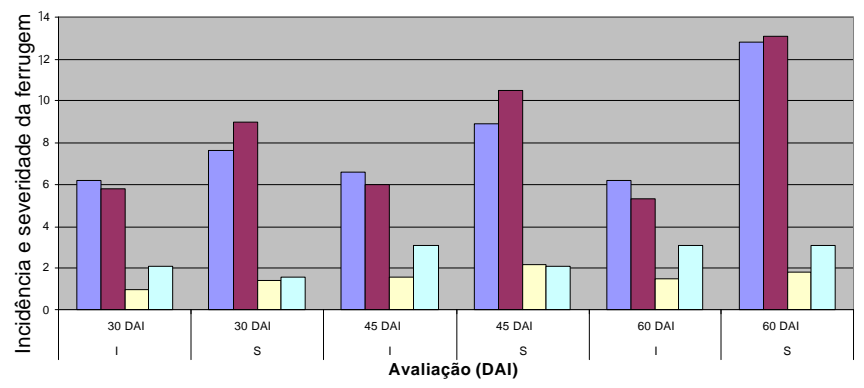

$\square$ Hidróxido de cobre sem chuva

$\square$ Hidróxido de cobre+óleo sem chuva

$\square$ Hidróxido de cobre com chuva

$\square$ Hidróxido de cobre+óleo com chuva

FIG. 2 - Comparativo de eficiência com fungicida a base de hidróxido de cobre, com e sem adição de óleo, na incidência (I) e severidade (S) da ferrugem (Hemileia vastatrix) em mudas de cafeeiros (Coffea arabica) tratadas, sob a influência ou não de chuva simulada.

na presença de chuvas semanais de $20 \mathrm{~mm}$ não foram significativas, apesar do seu melhor desempenho sem chuva.

Conclui-se que os cúpricos possuem ação diferenciada no controle da ferrugem do cafeeiro, e que o uso de adjuvantes à calda pode ser favorável ou não à tenacidade e à eficiência do produto, dependendo da formulação empregada. Os efeitos da intensidade da chuva e a adição de óleos devem ser analisados em conjunto e isoladamente para cada produto e formulação, envolvendo uma determinada superfície foliar e o tipo de patógeno.
TABELA 2 - Germinação in vitro de urediniosporos de Hemileia vastatrix obtidos em mudas de café (Coffea arabica) inoculadas e previamente tratadas com diferentes fungicidas cúpricos

\begin{tabular}{lcc}
\hline \multirow{2}{*}{ Tratamento } & \multicolumn{2}{c}{ \% de Germinação aos 62 DAI } \\
\cline { 2 - 3 } & Sem chuva & Com chuva \\
\hline 1- testemunha & $40,62 \mathrm{a}$ & $41,96 \mathrm{a}$ \\
2- óxido cuproso & 0,00 & $46,90 \mathrm{a}$ \\
3- óxido cuproso + óleo & $38,58 \mathrm{ab}$ & $41,30 \mathrm{a}$ \\
4- hidróxido de cobre & $44,80 \mathrm{a}$ & $44,65 \mathrm{a}$ \\
5- hidróxido de cobre + óleo & $35,39 \mathrm{ab}$ & $45,09 \mathrm{a}$ \\
6- oxicloreto de cobre & $35,84 \mathrm{ab}$ & $45,57 \mathrm{a}$ \\
7- oxicloreto de cobre + óleo & $14,43 \mathrm{c}$ & $12,60 \mathrm{c}$ \\
\hline CV\% & \multicolumn{3}{c}{12,3} \\
\hline
\end{tabular}

$\mathrm{SC}=$ sem chuva

$\mathrm{CC}=$ com chuva

Médias seguidas pela mesma letra, na vertical ou na horizontal, não diferem entre si pelo teste Tukey a $5 \%$ de probabilidade.



FIG. 3 - Comparativo de eficiência com fungicida a base de oxicloreto de cobre, com e sem adição de óleo, na incidência (I) e severidade (S) da ferrugem (Hemileia vastatrix) em mudas de cafeeiros (Coffea arabica) tratadas, sob a influência ou não de chuva simulada.

\section{LITERATURA CITADA}

ALMEIDA, S.R. \& MATIELLO, J.B. Efeito de fungicidas cúpricos e sistêmicos e sua associação para o controle de doenças (ferrugem e cercosporiose) no cafeeiro e sua ação sobre o desenvolvimento do sistema radicular e a produção. Anais, $25^{\circ}$ Congresso Brasileiro de Pesquisas Cafeeiras, Franca, SP. 1999. pp.16-17.

ALMEIDA, S.R. \& MATIELLO, J.B. Efeito de fungicidas cúpricos e sistêmicos e sua associação para o controle de doenças no cafeeiro e sua ação sobre o desenvolvimento do sistema radicular e a produção a médio prazo. In: Anais, $26^{\circ}$ Congresso Brasileiro de Pesquisas Cafeeiras, Marília, SP. 2000. pp.4850.

GODOY, C.V., BERGAMIN FILHO, A. \& SALGADO, C.L. 
Efeito da chuva sobre a tenacidade e eficiência de fungicidas...

Doenças do cafeeiro (Coffea arabica L.). In: Kimati, H. et al. (Eds.) Manual de Fitopatologia. Piracicaba. ESALQ. 1997. v.2. pp.184-200.

INSTITUTO BRASILEIRO DE CAFÉ. Cultura de Café no Brasil: manual de recomendações. 3 ed. Rio de Janeiro. Ed. Gerca. 1979.

KUSHALAPPA, A.C. \& ESKES, A.B. Coffee Rust: epidemiology, resistance and management. Boca Raton . CRC Press. 1989.

MATIELLO, J.B. O café do cultivo ao consumo. São Paulo, Globo Rural. 1991.

OLIVEIRA, S.H.F., TOFOLI, J.G. \& DOMINGUES, R.J. Persistência agronômica de mancozeb em diferentes concentrações de óleo vegetal e óleo mineral na cultura do trigo. Resumos, $18^{\circ}$ Congresso Paulista de Fitopatologia, Piracicaba, SP. 1995. p.21.

RICH, S. Dinamics of deposition and tenacity of fungicides. Phytopathology 44:203-213. 1954.

SANTINATO, R., AIZAWA, J.S., BECKER, A., FERNANDES, A.L. \& PEREIRA, E.M. Combinações de Cobre Sandoz, Recop e Resist com cyproconazole (Alto 100) via foliar no controle da ferrugem do cafeeiro alta. In: Anais, $25^{\circ}$ Congresso Brasileiro de Pesquisas Cafeeiras, Franca, SP. 1999. pp.255-257.

SOUZA, S.M.C. O café na região Sul de Minas Gerais - relação da qualidade com fatores ambientais, estruturais e tecnológicos. (Tese de Doutorado). Lavras. Universidade Federal de Lavras. 1996.

SUHERI, L. \& LATIN, C. Retention of fungicides for control of Alternaria leaf blight of muskmelon under geenhouse conditions. Plant Disease 75:1013-1015. 1991.

TOFOLI, J.G., OLIVEIRA, S.H.F. \& DOMINGUES, R.J. Efeito de diferentes concentrações de óleo vegetal na persistência agronômica de mancozeb em tomateiro. In: Resumos, $29^{\circ}$ Congresso Brasileiro de Fitopatologia, Itajaí, SC. 1996. p. 21.

TOFOLI, J.G., OLIVEIRA, S.H.F., DOMINGUES, R.J., SANTOS, J.M.F. \& MARTINS, E.M.S. Efeito da chuva sobre a sistemicidade, tenacidade e eficiência de azoxystrobin (Amistar $500 \mathrm{WG}$ ) no controle da ferugem (Hemileia vastatrix) em cafeeiro. In: Anais, $24^{\circ}$ Congresso Brasileiro de Pesquisas Cafeeiras, Poços de Caldas, MG. 1998. pp.180-182.

WEBER, A.L., McLEAN, H.C., DRIGGERS, B. F. \& ONEIL, W.J. Influence of different materials on coverage and adhesiveness of sprays and their effect on residue removal from apples. New Jersey Agricultural Experimental Station Bulletin 627:1-16. 1937.

ZAMBOLIM, L., DEL PELOSO, M.C. \& CHAVE, G.M. Principais doenças do cafeeiro. Informe Agropecuário 11:64-75. 1985.

ZAMBOLIM, L., VALE, F.X.R., CRUZ FILHO, J., CHAVES, G.M., CAIXETA, G. Z.T. \& MACABEU, A.J. Controle Químico da Ferrugem do Cafeeiro (Hemileia vastatrix) e Custos das Opções Propostas. Brasília. ABEAS. 1990.

ZAMBOLIN, L \& VALE, F.X.R. Perdas na produtividade e qualidade do cafeeiro causadas por doenças bióticas e abióticas. In: Zambolim, L (Ed.). Café - Produtividade, Qualidade e Sustentabilidade. Viçosa. Universidade Federal de Viçosa. 2000. pp.239-261. 